\title{
Long-Term Effects of Complex Decongestive Therapy in Breast Cancer Patients With Arm Lymphedema After Axillary Dissection
}

\author{
Jung Min Hwang, MD, Ji Hye Hwang, MD, Tae Won Kim, MD, \\ Seung Yeol Lee, MD, Hyun Ju Chang, PT, In Ho Chu, PT \\ Department of Physical and Rehabilitation Medicine, Samsung Medical Center, \\ Sungkyunkwan University School of Medicine, Seoul, Korea
}

\begin{abstract}
Objective To investigate the long-term effects of complex decongestive therapy (CDT) on edema reduction in breast cancer-related lymphedema patients after axillary dissection, according to the initial volume of edema. Methods A retrospective review of 57 patients with unilateral arm after an axillary dissection for breast cancer was performed. The patients, treated with two weeks of CDT and self-administered home therapy, were followed for 24 months. Arm volume was serially measured by using an optoelectronic volumeter prior to and immediately after CDT; and there were follow-up visits at 3, 6, 12, and 24 months. Patients were divided into two groups according to the percent excess volume (PEV) prior to CDT: group 1, $\mathrm{PEV}<20 \%$ and group 2, $\mathrm{PEV} \geq 20 \%$.

Results In group 1, mean PEV before CDT was $11.4 \pm 5.0 \%$ and $14.1 \pm 10.6 \%$ at 24 months after CDT with no significant difference. At the end of CDT, PEV was $28.8 \pm 15.7 \%$ in group 2, which was significantly lower than the baseline (41.9 $\pm 19.6 \%)$. The reduction of PEV was maintained for 24 months in group 2.

Conclusion The long-term effects of CDT were well-maintained for 24 months, but there was a difference in progression of PEV between the two groups. The patients with more initial PEV showed significant volumereducing effects of CDT. In patients with less initial PEV, the severity of lymphedema did not progress to higher grades.
\end{abstract}

Keywords Lymphedema, Physical therapy modalities, Breast neoplasms

\section{INTRODUCTION}

Received March 4, 2013; Accepted July 26, 2013

Corresponding author: Ji Hye Hwang

Department of Physical and Rehabilitation Medicine, Samsung Medical

Center, Sungkyunkwan University School of Medicine, 81 Irwon-ro, Gangnam-gu, Seoul 135-710, Korea

Tel: +82-2-3410-2816, Fax: +82-2-3410-0052, E-mail: hwanglee@skku.edu

(a) This is an open-access article distributed under the terms of the Creative Commons Attribution Non-Commercial License (http://creativecommons. org/licenses/by-nc/3.0) which permits unrestricted noncommercial use, distribution, and reproduction in any medium, provided the original work is properly cited.

Copyright $\odot 2013$ by Korean Academy of Rehabilitation Medicine

Arm lymphedema is well-established sequelae of breast cancer treatment. It causes functional and physical defects as well as psychological maladjustments, thereby reducing patient's quality of life [1-5]. Breast cancer-related lymphedema (BCRL) is affecting an increasing number of breast cancer patients with a longer survival rate for the patients [6]. Furthermore, patients who have undergone a mastectomy with axillary lymph node dissection (ALND) have a greater arm-volume and more commonly report 
symptoms of lymphedema, as compared to patients who have received a sentinel lymph node biopsy (SLNB) [5,7].

The major goal of lymphedema treatment is to reduce edema volume in the long-term and thus improve patient's quality of life. Current standard management of secondary arm lymphedema involves complex decongestive therapy (CDT). CDT includes the application of lowstretch bandaging, manual lymph drainage, compression therapy, exercise, and skin care [8].

Measurements of limb-volume may be used to monitor the progression of conditions as well as the response to $\mathrm{CDT}$. Some studies have reported volume reduction in limb lymphedema during the first 12 months or less of following CDT $[9,10]$, but there have been limited studies regarding the long-term effects of CDT $[11,12]$. Recently we reported that the long-term edema-reducing effects of CDT have been maintained over 24 months in lower-limb lymphedema [13]. To evaluate the continuous effects of CDT, it is important to discern, by follow-up examinations, the maintenance of long-term volume reduction.

Also, several studies have suggested that the initial volume of edema is the main key to a success in treatment, regardless of whether the treatment is early or late $[14,15]$. However, these studies did not show how long the beneficial effects of CDT lasted as based on the initial volume of edema. For this reason, we performed a retrospective assessment of the maintenance of volume-reduction in BCRL patients with ALND for 24 months after CDT. Patients were divided into two groups according to the percent excess volume (PEV) prior to $\mathrm{CDT}$, and we investigated the difference in change of PEV between the two groups.

The aim of this retrospective study was to investigate the long-term effects of CDT on edema reduction in BCRL patients after an axillary dissection, as well as to identify the difference in change of PEV according to the initial volume of edema.

\section{MATERIALS AND METHODS}

\section{Patients}

A retrospective chart review was performed for patients treated for arm lymphedema between January 1, 2001 and October 30, 2011. The inclusion criteria for this study were: 1) unilateral arm lymphedema after a mastectomy with ALND for breast cancer; 2) fully attended deconges- tive treatment five times per week for two weeks; and 3) clinical follow-up longer than two years after CDT. Patients who had a recurrence of cancer, other vascular, heart or renal disease and/or a past history of lymphedema treatment were excluded. Patients who underwent a mastectomy with SLNB had less severe lymphedema in the arm, which shortened the duration of CDT and resulted in insufficient follow-ups. For this reason, in our study, patients with SLNB were excluded. Also, patients who had an infection, trauma, and additional cancer treatment for 24 months were declined enrollment. Consequently, a total of 116 patients were eligible, of whom 57 (49\%) were enrolled for the study.

The following clinical characteristics were obtained after a retrospective chart review: patient's age, sex, body mass index [BMI], cancer-related therapy (type of surgery, chemotherapy, and radiotherapy), period of time from surgery to onset of lymphedema, chronicity of lymphedema, site of lymphedema (right or left), and lymphedema volume. This study was approved by the governing Institutional Review Board of our hospital.

\section{Complex decongestive therapy}

All patients were treated with CDT. The treatments were divided into two successive phases, which consisted of the decongestive and maintenance phase. In the decongestive phase, two certified skilled physical therapists performed two weeks of the CDT program at one single lymphedema center, five times a week. Each session of therapy included one hour of manual lymphatic drainage (MLD), compressive wrapping of the limb with multilayered and low-stretch compressive bandages, specific remedial exercises, and skin care $[11,16]$. This first decongestive phase aimed to obtain substantial volume reduction of the lymphedema [17].

After two weeks of the decongestive phase, patients followed a maintenance phase of self-care at home. Patients were taught self-bandaging, a self-massage technique, and remedial exercises under the supervision of a physical therapist. Patients were issued compression garments at the conclusion of the decongestive phase. The maintenance therapy consisted of daytime ready-made compression stockings, a daily session of self-administered MLD and skin care, as well as remedial exercises. Additionally, it was recommended that at least three overnight bandages be worn each week. During the maintenance 
phase, follow-up visits to outpatient clinics were scheduled at 3, 6, 12, and 24 months. We ascertained compliance with the maintenance therapy and encouraged the patients at each study time point.

\section{Limb volume measurements}

Lymphedema volume was serially measured prior to CDT, immediately after CDT, and at 3, 6, 12, and 24 months follow-up visits. Limb volume measurements were performed using an optoelectronic volumeter, Perometer (Pero-System, Wuppertal, Germany). Two measurements were obtained from the same site, and these measurements were then averaged. Measurements were taken by an examiner experienced in lymphedema treatment.

The therapeutic response of CDT was quantified as the change in the PEV of the treated limb. Excess volume was calculated as the difference in limb volume between the affected and unaffected sides [17]. The unaffected limb was used as a normal control for the affected limb. PEV was calculated using the following formula:

$$
\% \text { excess volume }=[(V a-V u) / V u] \times 100
$$

$V a$ represented the volume $(\mathrm{mL})$ of the affected upperlimb and $V u$ represented the volume $(\mathrm{mL})$ of the unaffected upper-limb. This parameter is better for evaluating edema evolution than absolute volume measurements, because of the heterogeneity of the anthropometric measures in the sample [15]. One method of establishing the severity of unilateral limb lymphedema is based on the difference in the limb volume of the affected and unaffected limbs. The International Society of Lymphology (ISL) [18] utilized the severity assessment based on the volume difference, assessed as minimal ( $<20 \%$ increase) in limb volume, moderate $(20 \%-40 \%$ increase $)$ or severe ( $>40 \%$ increase). In our study, patients were classified into two groups according to the PEV prior to CDT: group $1, \mathrm{PEV}<20 \%$ and group $2, \mathrm{PEV} \geq 20 \%$.

\section{Statistical analysis}

Descriptive statistics are presented for demographic and clinical/treatment factors of interest. The independent t-test was used to detect the significant difference in variables between the two groups. Between the two groups, the linear mixed model was used to detect the significant difference of the effects of CDT on lymphedema over time. Results were presented as means with standard deviations. The p-values less than 0.05 were considered as statistically significant. The collected data were analyzed using SPSS ver. 18.0 (SPSS Inc., Chicago, IL, USA).

\section{RESULTS}

\section{Descriptive characteristics}

A total of 57 patients were included in the study. Table 1 shows the baseline characteristics of the patients. Average age was $48.6 \pm 10.3$ years and one of the patients was a man. Among these patients, 34 patients $(59.6 \%)$ had lymphedema in the left arm and 23 patients $(40.3 \%)$ had lymphedema in the right arm. All of the patients had breast cancer surgery with axillary dissection: 46 patients $(80.7 \%)$ had a modified radical mastectomy (MRM) with ALND and 11 patients (19.3\%) had breast conserving surgery with ALND.

When the patients were classified according to the PEV prior to CDT, 32 patients $(56.1 \%)$ were placed in group 1 $(\mathrm{PEV}<20 \%)$ and 25 patients $(43.9 \%)$ were placed in group 2 ( $P E V \geq 20 \%$ ). The main comparisons of clinical characteristics between the two groups are presented in Table 2 . The average interval from the time of the surgery to the onset of lymphedema was approximately 19.3 months

Table 1. Patient characteristics $(n=57)$

\begin{tabular}{|cc|}
\hline Characteristic & No. of patients \\
\hline Age (yr), mean \pm SD & $48.6 \pm 10.3$ \\
\hline Sex (male:female) & $1: 56$ \\
\hline Site of lymphedema & \\
\hline Right & 23 \\
\hline Left & 34 \\
\hline Type of surgery & \\
\hline MRM with ALND & 46 \\
\hline BCS with ALND & 11 \\
\hline PEV prior to CDT & \\
\hline$<20 \%$ & 32 \\
$\geq 20 \%$ & 25 \\
\hline
\end{tabular}

SD, standard deviation; MRM, modified radical mastectomy; ALND, axillary lymph node dissection; BCS, breast conserving surgery; PEV, percent excess volume; CDT, complex decongestive therapy. 
Table 2. Comparisons between group 1 and group 2

\begin{tabular}{|lccc}
\hline \multicolumn{1}{c}{ Characteristic } & $\begin{array}{c}\text { Group 1 } \\
\text { PEV<20\% (n=32) }\end{array}$ & $\begin{array}{c}\text { Group 2 } \\
\text { PEV } \geq 20 \%(\mathbf{n}=\mathbf{2 5})\end{array}$ & p-value \\
\hline Age (yr) & $47.5 \pm 9.8$ & $50.1 \pm 10.9$ & 0.35 \\
\hline Body mass index $\left(\mathrm{kg} / \mathrm{m}^{2}\right)$ & $25.9 \pm 4.8$ & $26.1 \pm 4.4$ & 0.91 \\
\hline Time from surgery to lymphedema onset (mo) & $19.3 \pm 23.9$ & $32.2 \pm 46.8$ & 0.22 \\
\hline Chronicity of lymphedema (mo) & $8.5 \pm 6.6$ & $27.5 \pm 31.5$ & $0.006^{*}$ \\
\hline Chemotherapy (\%) & $24(75.0)$ & $17(68.0)$ & 0.56 \\
\hline Radiotherapy (\%) & $20(62.5)$ & $17(68.0)$ & 0.67 \\
MRM (\%) & $26(81.3)$ & $20(80.0)$ & 0.91 \\
PEV prior to CDT (\%) & $11.4 \pm 5.0$ & $41.9 \pm 19.6$ & $<0.001^{*}$ \\
\hline
\end{tabular}

Values are presented as mean \pm standard deviation or number (\%)

MRM, modified radical mastectomy; PEV, percent excess volume; CDT, complex decongestive therapy.

${ }^{*} \mathrm{p}<0.05$.

Table 3. PEV (\%) at study time points

\begin{tabular}{lcc}
\hline & $\begin{array}{c}\text { Group 1 } \\
\text { PEV }<\mathbf{2 0 \%}(\mathbf{n}=\mathbf{3 2})\end{array}$ & $\begin{array}{c}\text { Group 2 } \\
\text { PEV } \geq \mathbf{2 0 \%}(\mathbf{n}=\mathbf{2 5})\end{array}$ \\
\hline Pre-CDT & $11.4 \pm 5.0$ & $41.9 \pm 19.6$ \\
Post-CDT & $10.2 \pm 8.9$ & $28.8 \pm 15.7$ \\
3-mo follow-up & $10.4 \pm 7.4$ & $28.3 \pm 12.0$ \\
6-mo follow-up & $11.7 \pm 7.7$ & $20.5 \pm 32.3$ \\
1-yr follow-up & $13.1 \pm 9.8$ & $25.5 \pm 12.9$ \\
2-yr follow-up & $14.1 \pm 10.6$ & $27.0 \pm 18.0$ \\
\hline
\end{tabular}

PEV, percent excess volume; CDT, complex decongestive therapy; pre-CDT, prior to CDT; post-CDT, right after CDT.

in group 1 and 32.2 months in group 2 and did not differ significantly in each group $(\mathrm{p}=0.22)$. However between the two groups, the chronicity of lymphedema was significantly different statistically ( $\mathrm{p}=0.006$ ). In group 1 , the chronicity of lymphedema was $8.5 \pm 6.6$ months; and in group 2, it was $27.5 \pm 31.5$ months.

There was no significant statistical difference between each group in age $(\mathrm{p}=0.35), \mathrm{BMI}(\mathrm{p}=0.91)$, chemotherapy $(\mathrm{p}=0.56)$, radiotherapy $(\mathrm{p}=0.67)$, and MRM $(\mathrm{p}=0.91)$. The mean PEV before CDT was $11.4 \pm 5.0 \%$ in group 1 and $41.9 \pm 19.6 \%$ in group 2 . PEV before CDT in group 2 was significantly higher statistically than that in group 1. According to the ISL definition, the baseline severity of group 1 was minimal lymphedema, and the baseline severity of group 2 was severe lymphedema. Stillwell [19] defined "slight" lymphedema as PEV of $11 \%-20 \%$, and PEV below $10 \%$ he called "insignificant". In group 1, the baseline PEV was $11.4 \%$, which was slight lymphedema

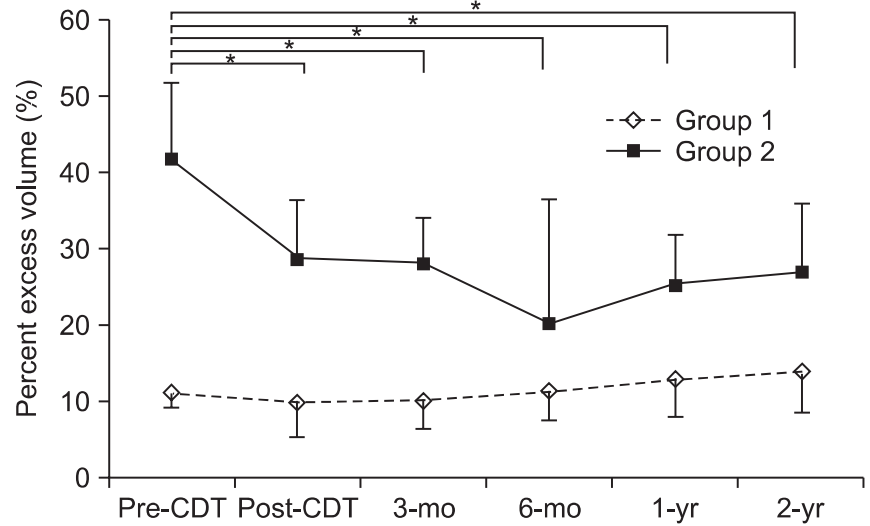

Fig. 1. Distribution of percent excess volume (PEV) at study time points. At months 3, 6, 12, and 24 after CDT, the PEV in group 2 (PEV $\geq 20 \%)$ was significantly lower than at the baseline, but no significant difference was seen in group $1(\mathrm{PEV}<20 \%)$. Error bars indicate a standard deviation. CDT, complex decongestive therapy; pre$\mathrm{CDT}$, prior to CDT; post-CDT, right after CDT. ${ }^{*} \mathrm{p}<0.05$, comparison with pre-CDT.

according to this classification.

\section{Percent excess volume}

When compared to the unaffected arm, data for the mean PEV changes of the affected arm before CDT (preCDT), right after CDT (post-CDT) and at 3, 6, 12, and 24 months after CDT are shown in Table 3. There was a decrease in PEV from pre-CDT to post-CDT, 3, 6, 12, and 24 months after CDT in group 2. The greatest decrease in PEV was at 6 months after CDT, with PEV tending to increase over time. At post-CDT and 3, 6, 12, and 24 months after CDT, the PEV in group 2 was significantly lower than 
pre-CDT ( $\mathrm{p}<0.001)$ (Fig. 1).

In group 1 , there was a decrease in PEV from pre-CDT to post-CDT and 3 months after CDT. At 6,12 , and 24 months after CDT, the PEV started to increase. However, during the maintenance phase, there were no significant increases in PEV compared with pre-CDT (Fig. 1).

\section{DISCUSSION}

This study investigated long-term effects of CDT on edema reduction in patients who suffered from BCRL after an axillary dissection. The patients were classified into two groups according to the volume of edema prior to CDT. The different outcomes of CDT for the two groups were investigated.

Earlier studies have shown various factors influencing the volume of BCRL after CDT [20]. The different effects of CDT may be related to the quality of CDT as well as the different diagnostic methods used for the limb-volume measurement. In this study, we attempted to control the factors that might influence the results of CDT. All patients were recruited in a single clinic by a clinician, and the treatments were homogeneous. Limb volume measurements were performed using an optoelectronic volumeter, which was able to overcome many of the limitations of the traditional diagnostic tools [21]. The optoelectronic volumeter has been found to have increased sensitivity and decreased variability in measuring BCRL [22]. Also, optoelectronic volumeter allows for a fast and relatively accurate measurement of arm volume and facilitates routine monitoring of arm volume in clinical practice.

Lymphedema is very frequently quantified as an absolute volume-change in the literature [20]. However, absolute volume measurements lack specificity, because the magnitude of absolute volume change depends on the body size (weight and BMI) of each patient [23]. A relative volume difference between the affected and unaffected arms expressed as a percent change is a more reliable method, as such does not vary with body size and shape [23]. There has been an increasing number of studies using the relative difference of arm volumes to minimize the effect of body weight $[15,24,25]$. Ancukiewicz et al. [23] conducted a prospective study of 677 patients with breast cancer and reported that temporal changes in the absolute volume of the unaffected arm is correlated with patient body size, while relative arm volume change does not. We used PEV, not absolute excess volume, to calculate lymphedema severity, so as to exclude the confounding factor of individual body size. However, some studies have found that BMI is correlated with CDT efficacy and lymphedema severity $[11,12,26]$. Hence it is a limitation of this study that the temporal change of BMI is not recorded. In some cases, absolute volume of the unaffected arm increased as compared to that before CDT; or absolute volume of the affected arm decreased compared to the unaffected. These results might be due to an increased activity of the unaffected arm, fibrosis or atrophy of the affected, and disuse of the affected [27]. As there are individual variations in the unilateral arm, relative volume measurement may be complemented by the absolute volume measurement.

Another point to consider when comparing the volume of affected limb with the unaffected contralateral limb is the natural difference in limb size, especially the dominant versus the non-dominant arm. The mean normal inter-limb difference is known to be $3 \%$ [28]. In this retrospective study, we could not analyze the volume change according to the hand dominance. However, we diagnosed BCRL with a combined method of volume measurement and physical examination, which might enable proper exclusion of the normal inter-limb differences. As there are individual variations in the normal inter-limb differences, the assessment of volume change must be supplemented with qualitative evaluation to make a sensitive diagnosis of BCRL [24].

When the two groups of the study were compared, it was found that there was no significant statistical difference between the patients of varying age, BMI, the time of surgery to onset of lymphedema, chemotherapy, radiotherapy, and MRM. The groups were relatively homogenous. However, the chronicity of lymphedema was significantly different between the two groups $(p=0.006)$ (Table 2). The patients of group 2 have had chronic lymphedema for more than two years, with significantly higher initial PEV ( $\mathrm{p}<0.001)$ (Table 2). This was compatible with results of other studies, in which the amount of initial swelling was correlated with lymphedema duration $[12,29]$.

In previous studies, CDT has been shown to be effective in reducing edema, both in acute as well as chronic cases. Vignes et al. [12] found that the chronicity of 
lymphedema was a predictive factor of response when the response was measured in the absolute value of reduction in lymphedema volume; but relative percentage of volume-reduction was same, regardless of the chronicity. Moreover, in many other studies, the chronicity of lymphedema was not a predictive factor of response $[11,14,15,29,30]$. Based on these studies, there seems to be a low-correlation between the chronicity and CDT outcome.

Although there was no significant volume reduction in group 1, slight lymphedema was not worsened to moderate or severe lymphedema for 24 months. According to a previous study [31], a progression of untreated lymphedema is common. Similarly, Bar Ad et al. [32] analyzed the time-course of arm lymphedema after breast conservation treatment and showed that one-third of patients with mild and moderate arm lymphedema at the time of initial diagnosis progressed to a higher grade during the first 5 years of follow-up after the initial arm lymphedema development. These results may be compared to the results of our study, with no progression to a higher grade in group 1 . No progression to higher grades during 24 months may be a meaningful effect of CDT in group 1 .

Previous studies $[14,15,33]$ have reported that patients with lower PEV showed a better response to CDT, but such does not correspond with our results. However, the mean initial PEV of these studies was 35.3\% [15] and 27.7\% [33]. These initial PEV were moderate lymphedema according to the ISL definition [18] and markedly different from that of group 1 (11.4\%). Also, they measured the arm volume with circumference, which is less accurate than optoelectronic volumeter as used in our study. Also these studies did not perform long-term follow-ups to examine the beneficial effects of CDT.

To our knowledge, there are limited reports concerning the CDT response for slight lymphedema. Our results in group 1 were supported by a retrospective study [24] of 98 patients with BCRL with a follow-up of 10 years, where the mean PEV was $8.1 \%$ at diagnosis and $9.0 \%$ at the last follow-up measurement (mean 48.9 months), with no significant difference. Our results of group 1 were similar to the results of this previous study. In group 1, at 6 months after CDT, PEV was observed to increase again with no significant difference as compared to the initial PEV (Table 3). An explanation for this increase after 6 months might be that there were cases of localized edema in group 1. Lymphedema is not necessarily uniform in distribution and may instead develop in a localized arm segment. Localized swelling may precede generalized arm lymphedema. However, current methods of assessing lymphedema based on the whole-limb measures may not detect these early localized changes [20]. Therefore, additional research is required to assess the localized swelling in mild lymphedema, and improved techniques are needed to examine the localized edema.

In group 2, PEV improved from $41.9 \%$ to $28.8 \%$ after $\mathrm{CDT}$, or from severe lymphedema to moderate lymphedema. At 24 months after CDT, PEV was $27.0 \%$ and reduced to $64.4 \%$ compared to pre-CDT $(41.9 \%)$. These positive effects of CDT coincide with previous findings of CDT reducing lymphatic volume [34-36]. Fig. 1 shows the similarity between PEV at post-CDT and PEV at 24 months, suggesting that PEV at post-CDT may be used to predict a long-term outcome. Although there is no improvement to $\mathrm{PEV}<20 \%$ (mild lymphedema) during the follow-up period, it may be argued that BCRL with a large initial PEV can be reduced to about half the amount by CDT. Hence we should encourage patients to receive CDT even with severe lymphedema.

Several limitations of this study warrant discussion. First, because we enrolled only $49 \%$ of eligible patients, our findings must be generalized cautiously. Second, since ours was a retrospective analysis, we did not formally assess the fidelity of the patients to the maintenance therapy guidelines, similarly to other retrospective studies $[24,30,33,35,37]$. However, since patients' compliance to CDT is an important predictor of response [15], further studies are needed to compare different compliance with stratified analysis. Third, the qualitative evaluation of dermal thickness, fibrosis and skin tone can contribute to confirmation of the clinical diagnosis of lymphedema. However, we did not perform qualitative evaluation of these signs. Those patients with chronic lymphedema especially may have extensive fibrosis in the tissues, and they may exhibit less effects of CDT. Therefore, evaluation of fibrosis tends to a more precise assessment of CDT response. However, to our knowledge, the methods of measuring fibrosis have not been tested for validity or reliability $[10,15,30,38]$. Previous studies $[11,12,14,33]$ have not assessed the presence of fibrosis, and an explanation for the relationship between fibrosis and CDT response cannot be found. Although there is a study [15] which 
reported that fibrosis is not a predictive factor of CDT response, the study does not specify methods of measuring fibrosis. Therefore, further study is required to validate the qualitative evaluation of tissue character and skin condition with additional instruments, such as ultrasound, tissue tonometer, and bioimpedance. Also, based on the establishment of methods, additional research is required to understand the relationship between fibrosis and CDT response.

In the long-term (over 24 months), CDT was found to be effective in BCRL after a mastectomy with ALND, but there was a difference in progression of PEV according to the initial PEV. Although there was no improvement to $\mathrm{PEV}<20 \%$, patients with more initial PEV showed significant volume-reducing effects of CDT. On the other hand, the severity of lymphedema did not progress to higher grades in patients with less initial PEV, although there was no significant volume difference for 24 months. Therefore, it is important to encourage patients to receive CDT, both in mild and severe lymphedema. Several limitations notwithstanding, the results of our study may help guide management in the long-term care of BCRL patients after axillary dissection.

\section{CONFLICT OF INTEREST}

No potential conflict of interest relevant to this article was reported.

\section{REFERENCES}

1. Ahmed RL, Prizment A, Lazovich D, Schmitz KH, Folsom AR. Lymphedema and quality of life in breast cancer survivors: the Iowa Women's Health Study. J Clin Oncol 2008;26:5689-96.

2. Pusic AL, Cemal Y, Albornoz C, Klassen A, Cano S, Sulimanoff I, et al. Quality of life among breast cancer patients with lymphedema: a systematic review of patient-reported outcome instruments and outcomes. J Cancer Surviv 2013;7:83-92.

3. Shah C, Vicini FA. Breast cancer-related arm lymphedema: incidence rates, diagnostic techniques, optimal management and risk reduction strategies. Int J Radiat Oncol Biol Phys 2011;81:907-14.

4. Gartner R, Jensen MB, Kronborg L, Ewertz M, Kehlet $\mathrm{H}$, Kroman N. Self-reported arm-lymphedema and functional impairment after breast cancer treatment: a nationwide study of prevalence and associated factors. Breast 2010;19:506-15.

5. Ashikaga T, Krag DN, Land SR, Julian TB, Anderson SJ, Brown AM, et al. Morbidity results from the NSABP B-32 trial comparing sentinel lymph node dissection versus axillary dissection. J Surg Oncol 2010;102:1118.

6. Mouridsen HT, Bjerre KD, Christiansen P, Jensen MB, Moller S. Improvement of prognosis in breast cancer in Denmark 1977-2006, based on the nationwide reporting to the DBCG Registry. Acta Oncol 2008;47:52536.

7. Miller CL, Specht MC, Skolny MN, Jammallo LS, Horick N, O'Toole J, et al. Sentinel lymph node biopsy at the time of mastectomy does not increase the risk of lymphedema: implications for prophylactic surgery. Breast Cancer Res Treat 2012;135:781-9.

8. Foldi E, Foldi M, Clodius L. The lymphedema chaos: a lancet. Ann Plast Surg 1989;22:505-15.

9. Vignes S, Porcher R, Arrault M, Dupuy A. Long-term management of breast cancer-related lymphedema after intensive decongestive physiotherapy. Breast Cancer Res Treat 2007;101:285-90.

10. Ko DS, Lerner R, Klose G, Cosimi AB. Effective treatment of lymphedema of the extremities. Arch Surg 1998;133:452-8

11. Vignes S, Porcher R, Arrault M, Dupuy A. Factors influencing breast cancer-related lymphedema volume after intensive decongestive physiotherapy. Support Care Cancer 2011;19:935-40.

12. Vignes S, Porcher R, Champagne A, Dupuy A. Predictive factors of response to intensive decongestive physiotherapy in upper limb lymphedema after breast cancer treatment: a cohort study. Breast Cancer Res Treat 2006;98:1-6.

13. Kim YB, Hwang JH, Kim TW, Chang HJ, Lee SG. Would complex decongestive therapy reveal long term effect and lymphoscintigraphy predict the outcome of lower-limb lymphedema related to gynecologic cancer treatment? Gynecol Oncol 2012;127:638-42.

14. Ramos SM, O'Donnell LS, Knight G. Edema volume, not timing, is the key to success in lymphedema treatment. Am J Surg 1999;178:311-5.

15. Forner-Cordero I, Munoz-Langa J, Forner-Cordero A, De Miguel-Jimeno JM. Predictive factors of re- 
sponse to decongestive therapy in patients with breast-cancer-related lymphedema. Ann Surg Oncol 2010;17:744-51.

16. Bernas MJ, Witte CL, Witte MH; International Society of Lymphology Executive Committee. The diagnosis and treatment of peripheral lymphedema: draft revision of the 1995 Consensus Document of the International Society of Lymphology Executive Committee for discussion at the September 3-7, 2001, XVIII International Congress of Lymphology in Genoa, Italy. Lymphology 2001;34:84-91.

17. Szuba A, Cooke JP, Yousuf S, Rockson SG. Decongestive lymphatic therapy for patients with cancer-related or primary lymphedema. Am J Med 2000;109:296-300.

18. Piller N, Carati C. The diagnosis and treatment of peripheral lymphedema. Lymphology 2009;42:146-7.

19. Stillwell GK. Treatment of postmastectomy lymphedema. Mod Treat 1969;6:396-412.

20. Lasinski BB, McKillip Thrift K, Squire D, Austin MK, Smith KM, Wanchai A, et al. A systematic review of the evidence for complete decongestive therapy in the treatment of lymphedema from 2004 to 2011. PM R 2012;4:580-601.

21. Stanton AW, Northfield JW, Holroyd B, Mortimer PS, Levick JR. Validation of an optoelectronic limb volumeter (Perometer). Lymphology 1997;30:77-97.

22. Tierney S, Aslam M, Rennie K, Grace P. Infrared optoelectronic volumetry, the ideal way to measure limb volume. Eur J Vasc Endovasc Surg 1996;12:412-7.

23. Ancukiewicz M, Miller CL, Skolny MN, O’ Toole J, Warren LE, Jammallo LS, et al. Comparison of relative versus absolute arm size change as criteria for quantifying breast cancer-related lymphedema: the flaws in current studies and need for universal methodology. Breast Cancer Res Treat 2012;135:145-52.

24. Johansson K, Branje E. Arm lymphoedema in a cohort of breast cancer survivors 10 years after diagnosis. Acta Oncol 2010;49:166-73.

25. O'Toole J, Jammallo LS, Skolny MN, Miller CL, Elliott $\mathrm{K}$, Specht MC, et al. Lymphedema following treatment for breast cancer: a new approach to an old problem. Crit Rev Oncol Hematol 2013 Jun 15 [E-pub]. http:// dx.doi.org/10.1016/j.critrevonc.2013.05.001.

26. Helyer LK, Varnic M, Le LW, Leong W, McCready D. Obesity is a risk factor for developing postoperative lymphedema in breast cancer patients. Breast J 2010;16:48-54.
27. Sitzia J, Stanton AW, Badger C. A review of outcome indicators in the treatment of chronic limb oedema. Clin Rehabil 1997;11:181-91.

28. Gebruers N, Truijen S, Engelborghs S, De Deyn PP. Volumetric evaluation of upper extremities in 250 healthy persons. Clin Physiol Funct Imaging 2007;27:17-22.

29. Liao SF, Huang MS, Li SH, Chen IR, Wei TS, Kuo SJ, et al. Complex decongestive physiotherapy for patients with chronic cancer-associated lymphedema. J Formos Med Assoc 2004;103:344-8.

30. Yamamoto R, Yamamoto T. Effectiveness of the treatment-phase of two-phase complex decongestive physiotherapy for the treatment of extremity lymphedema. Int J Clin Oncol 2007;12:463-8.

31. Casley-Smith JR. Alterations of untreated lymphedema and it's grades over time. Lymphology 1995; 28:174-85.

32. Bar Ad V, Dutta PR, Solin LJ, Hwang WT, Tan KS, Both $\mathrm{S}$, et al. Time-course of arm lymphedema and potential risk factors for progression of lymphedema after breast conservation treatment for early stage breast cancer. Breast J 2012;18:219-25.

33. Liao SF, Li SH, Huang HY, Chen ST, Kuo SJ, Chen DR, et al. The efficacy of complex decongestive physiotherapy (CDP) and predictive factors of lymphedema severity and response to CDP in breast cancer-related lymphedema (BCRL). Breast 2013;22:703-6.

34. Cheifetz O, Haley L; Breast Cancer Action. Management of secondary lymphedema related to breast cancer. Can Fam Physician 2010;56:1277-84.

35. Hamner JB, Fleming MD. Lymphedema therapy reduces the volume of edema and pain in patients with breast cancer. Ann Surg Oncol 2007;14:1904-8.

36. Korpan MI, Crevenna R, Fialka-Moser V. Lymphedema: a therapeutic approach in the treatment and rehabilitation of cancer patients. Am J Phys Med Rehabil 2011;90:S69-75.

37. Jeffs E. Treating breast cancer-related lymphoedema at the London Haven: clinical audit results. Eur J Oncol Nurs 2006;10:71-9.

38. Williams AF, Vadgama A, Franks PJ, Mortimer PS. A randomized controlled crossover study of manual lymphatic drainage therapy in women with breast cancer-related lymphoedema. Eur J Cancer Care (Engl) 2002;11:254-61. 